\title{
Suggested standards for reporting power and energy density in supercapacitor research
}

\author{
Y HU and T S FISHER* D \\ Department of Mechanical \& Aerospace Engineering and California nanoSystems Institute, University of California, \\ Los Angeles, CA 90095, USA \\ *Author for correspondence (tsfisher@ucla.edu)
}

MS received 15 March 2018; accepted 7 May 2018; published online 6 September 2018

\begin{abstract}
One of the most important applications for porous material with high surface area is the supercapacitor. For supercapacitors, power density and energy density are the most significant metrics. However, currently no dominant, established standard exists, and research papers often report these two metrics differently. A standard for reporting power and energy density is proposed here for objective comparison. This paper also discusses a common flaw in current supercapacitor power density calculations.
\end{abstract}

Keywords. Supercapacitor; power density; energy density.

\section{Introduction}

Supercapacitors, with high power and energy density, long cycle life and low minimum working temperature, are widely used in hybrid vehicles, emergency electrical back-up and engine starters for low-temperature applications [1]. The growing supercapacitor market stimulates researchers to develop robust and high-surface-area materials as electrodes that enable high power density and energy density.

Based on Google scholar data, more than 20,000 papers have been published on supercapacitors since 2014, and continuously increasing power and energy density have been reported. However, on scrutinizing published data, some inconsistencies are apparent between different papers. First, multiple methods exist for capacitance and equivalent series resistance (ESR) measurements. The most widely used techniques are three-electrode measurements for testing a single-electrode sample and two-electrode techniques for sandwiched electrodes. For the same sample, the threeelectrode measurement yields twice the capacitance and half the ESR compared with two-electrode measurements. Occasionally, the method used is omitted from the reporting. Second, the bases upon which power and energy density are computed are sometimes arbitrarily chosen [2]. The commonly used bases are mass or volume of active material and mass or volume of electrode. Different bases yield orders of magnitude differences in power and energy density, yet it is not uncommon to omit the details of the bases. Third, different researchers use different scan rates for capacitance measurement. Typically, lower scan rate yields higher capacitance and thereby higher energy density, especially for supercapacitors with pseudocapacitance. However, this should be avoided since supercapacitors typically work at very high rate of charge and discharge, as pointed out in a previous article of the field [3]. Therefore, a universal standard for reporting power and energy density is required to compare research work fairly and reduce the confusion of calculations and comparisons.

\section{A proposed standard for reporting power and energy density}

In contrast with laboratory devices, comparing power and energy density of commercial supercapacitor modules is convenient, since most of them have the same output voltage (typically $16 \mathrm{~V}$ for DC applications) and the dimension and weight of the supercapacitor modules are available. Similarly, to make fair comparisons of emerging laboratory prototypes, the power and energy density of the final product (supercapacitor modules with the same output voltage) should be evaluated. Typically, such a requirement is difficult to fulfil for research laboratories that often lack final packaging and integration solutions. Therefore, this approach suggested here 'virtually' performs the assembly process of supercapacitors and computes the power and energy density of the 'finished' supercapacitor module.

The assembly process consists of three steps as shown in figure 1. First, a unit electrode sample with known properties (ESR, capacitance and maximum voltage) is obtained. Many such unit electrodes are connected to increase surface area to achieve lower ESR and higher capacitance, as shown in figure 1A [4] and 1B [5]. Second, two electrodes from the first step are sandwiched to form a supercapacitor device, as shown in figure 1B. The last step is to connect supercapacitors 

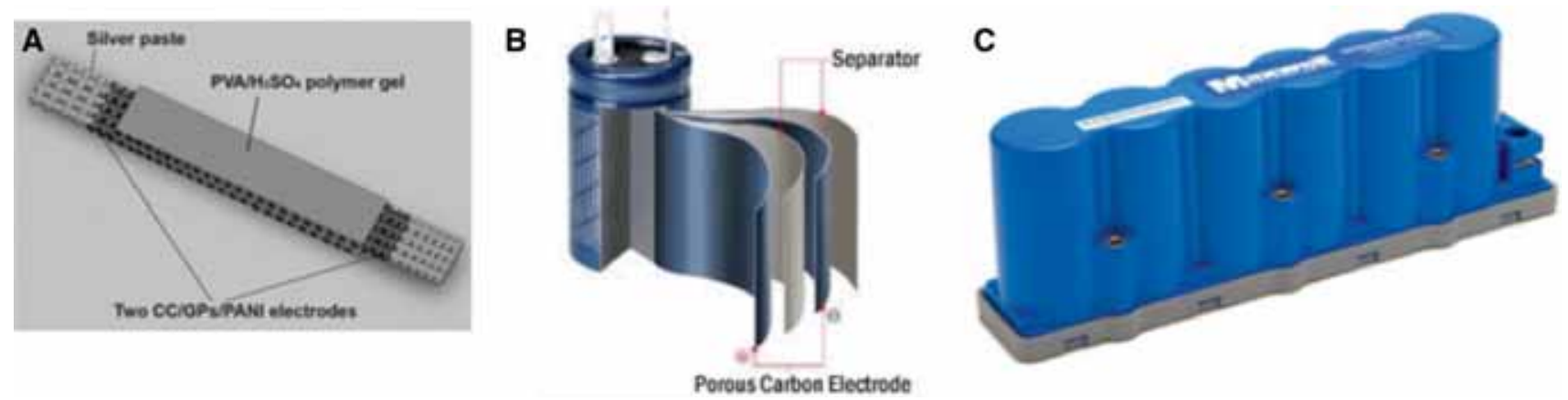

Figure 1. Supercapacitor module assembly process, reprinted with permission [4-6].

from step 2 in series as a supercapacitor module to achieve a given output voltage, as demonstrated in figure $1 \mathrm{C}[6]$.

\subsection{Power and energy density for electrode samples measured with three-electrode set-ups}

2.1a Extend electrode surface area: We first assume that the properties of a unit electrode are measured using threeelectrode set-up with ESR $R_{0}$, capacitance $C_{0}$ and maximum working voltage $V_{0}$. The extensive property of the unit electrode, either mass or volume, is $B_{0} . Z_{0}$ is the impedance of the unit electrode, $\omega$ is the angular frequency and $j$ is the imaginary unit number. In this work, the supercapacitor is considered to be an ideal capacitor connected in series with an equivalent series resistor. However, most double-layer supercapacitors are represented very well by De Levie's model [7], which is essentially an infinitely parallel connection of units consisting of series connections of an ideal resistor and an ideal capacitor. Therefore, the result derived in this section is also applicable to a double-layer supercapacitor.

The first step is to create a larger electrode from the sample electrode to increase capacitance as well as to reduce ESR. Assuming that the extensive property of this larger electrode (subscript 1 ) is $B_{1}$, the impedance is

$$
\begin{aligned}
Z_{0} & =R_{0}+\frac{1}{j C_{0} \omega}, \\
Z_{1}^{-1} & =\frac{B_{1}}{B_{0}} Z_{0}^{-1}, \\
Z_{1} & =\frac{B_{0}}{B_{1}} R_{0}+\frac{1}{j\left(B_{1} / B_{0} C\right) \omega} .
\end{aligned}
$$

Therefore, the ESR is $R_{0} B_{0} / B_{1}$ and the capacitance is $C_{0} B_{1} / B_{0}$. The maximum voltage does not change because it is determined by the electrolyte.

2.1b Extend electrode surface area: The second step is to construct a full-cell supercapacitor device (subscript 2) using the electrode obtained in step 1:

$$
Z_{2}=Z_{1}+Z_{1}=2 Z_{1}=\frac{2 B_{0}}{B_{1}} R_{0}+\frac{1}{j\left(C B_{1} /\left(2 B_{0}\right)\right) \omega} .
$$

Therefore, the ESR is $2 B_{0} R_{0} / B_{1}$, and the capacitance is $C_{0} B_{1} / 2 B_{0}$, while the maximum voltage remains the same. The extensive property is $B_{2}=2 B_{1}$, because two individual electrodes are required to build a full cell.

2.1c Assemble into modules: The last step is to assemble the supercapacitor device from step 2 and form a supercapacitor module (subscript 3 ) to achieve the target voltage $V_{3}$. To achieve this goal, the supercapacitor devices must be connected in series, and the number of such device required is $V_{3} / V_{1}$.

$$
\begin{aligned}
Z_{3} & =\frac{B_{3}}{B_{2}} Z_{2}=\frac{B_{3}}{B_{2}} R_{2}+\frac{1}{j\left(B_{2} C_{2} / B_{3}\right) \omega} \\
& =\frac{4 B_{3}}{B_{0}} R_{0}+\frac{1}{j\left(B_{0} C_{0} / 4 B_{3}\right) \omega} .
\end{aligned}
$$

ESR of the assembled module is obtained by setting $w$ to infinite in equation (5). Therefore, the power density of the supercapacitor module is

$$
\begin{aligned}
\frac{P_{3}}{B_{3}} & =\frac{V_{3}^{2}}{4 R_{3} B_{3}}=\frac{V_{3}^{2}}{4\left(4 B_{3} / B_{0}\right) R_{0} B_{3}}=\frac{\left(V_{0}^{2} B_{3}^{2} / B_{0}^{2}\right)}{16 B_{3}^{2} R_{0} / B_{0}} \\
& =\frac{V_{0}^{2}}{16 R_{0} B_{0}} .
\end{aligned}
$$

Similarly, for energy density

$$
\frac{E_{3}}{B_{3}}=\frac{C_{3} V_{3}^{2}}{2 B_{3}}=\frac{\left(B_{0} C_{0} / 4 B_{3}\right)\left(V_{0}^{2} B_{3}^{2} / B_{0}^{2}\right)}{2 B_{3}}=\frac{C_{0} V_{0}^{2}}{8 B_{0}} .
$$

As shown in equations (6) and (7), the power and energy densities of the supercapacitor module depend only on the properties of the unit electrode sample. Therefore, in addition to reporting ESR and capacitance of the sample (using a three-electrode set-up), the extensive property of the electrode (mass and volume) must also be reported. 


\subsection{Power and energy density for electrode samples measured with two-electrode set-ups}

Some researchers prefer reporting the result of sandwiched electrodes using a two-electrode set-up. Here, we assume that ESR is $R_{\mathrm{f}}$, capacitance is $C_{\mathrm{f}}$ and maximum working voltage is $V_{\mathrm{f}}$, and the extensive property of the device is $B_{\mathrm{f}}$. The full device is assembled into a supercapacitor module with output voltage of $V_{3}$ and extensive property $B_{3}$. Exactly the same calculation steps yield the following results for energy and power density:

$$
\begin{aligned}
& \frac{P_{3}}{B_{3}}=\frac{V_{\mathrm{f}}^{2}}{4 R_{\mathrm{f}} B_{\mathrm{f}}}, \\
& \frac{E_{3}}{B_{3}}=\frac{C_{\mathrm{f}} V_{\mathrm{f}}^{2}}{2 B_{\mathrm{f}}} .
\end{aligned}
$$

\subsection{Summary}

To summarize, power and energy density can be obtained to make objective comparisons as follows:

$$
\begin{aligned}
& \frac{P}{B}= \begin{cases}\left(V_{0}^{2} / 16 R_{0} B_{0}\right), & \text { three-electrode setup } \\
\left(V_{\mathrm{f}}^{2} / 4 R_{\mathrm{f}} B_{\mathrm{f}}\right), & \text { two-electrode setup }\end{cases} \\
& \frac{E}{B}= \begin{cases}\left(C_{0} V_{0}^{2} / 8 B_{0}\right), & \text { three-electrode setup } \\
\left(C_{\mathrm{f}} V_{\mathrm{f}}^{2} / 2 B_{\mathrm{f}}\right), & \text { two-electrode setup }\end{cases}
\end{aligned}
$$

Both results depend only on the intrinsic properties of the unit electrode or unit supercapacitor cell. The results also emphasize the importance of reporting the appropriate extensive property of the unit electrode or the unit supercapacitor cell; preferably both mass and volume should be reported.

Based on equations (10) and (11), power and energy densities of a few widely used commercial supercapacitors [8-10] have been calculated. Some published results [11-13] where power and energy densities are calculated based on device volume are also presented in figure 2. All the listed commercial supercapacitors exhibit higher energy density, and typically higher power density. The primary reason is that laboratory supercapacitors tend to be more amenable to thin-film formats, as opposed to bulk, professionally packaged capacitors. Nevertheless, improvement in power and energy density is required to compete with commercial supercapacitors.

\section{Discussion of a widely used power density calculation method}

The generally accepted approach to calculate supercapacitor power is [14]

$$
P=V^{2} / 4 R
$$

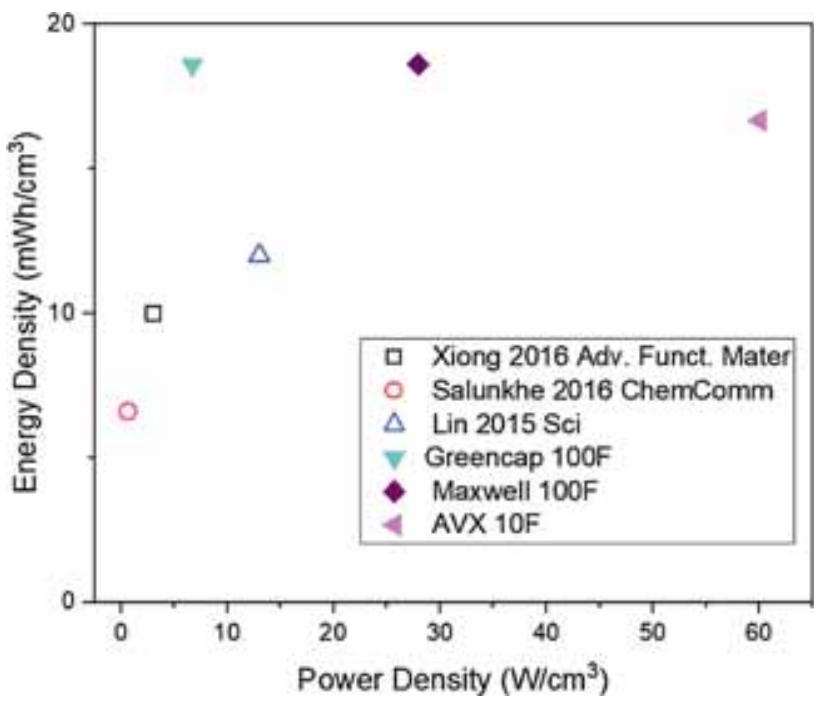

Figure 2. Ragone plot for commercial supercapacitors [8-10] as well as some published results [11-13]. The power and energy densities are calculated based on equations (10) and (11).

where $R$ represents the ESR. This approach computes the maximum possible power distributed to the load, when the resistance of the load matches the ESR of the supercapacitor. Another common approach in literature $[11,15,16]$ for power density calculations is

$$
\begin{aligned}
& P=E / \Delta t \\
& E=C V^{2} / 2 B,
\end{aligned}
$$

where $V$ is the applied voltage, $C$ the capacitance of the supercapacitor, $B$ the extensive property of the electrode and $\Delta t$ the discharge time under constant current.

The flaw with using this approach is that, because the supercapacitor is discharged using a galvanostatic instrument, the discharge time does not always reflect the true currentcarrying capability of the supercapacitor being tested. For galvanostatic discharge, the discharge time is

$$
\Delta t=\frac{V}{I / C}
$$

where $I$ is the galvanostatic discharge current. According to equations (13) and (14)

$$
P=\frac{E}{\Delta t}=\frac{C V^{2} I}{2 B V C}=\frac{V I}{2 B}
$$

The power density computed from this approach therefore depends on the current setting of galvanostatic instrument. If the current is set to higher than the shorting current, the power density obtained using equation (16) exceeds the maximum possible power output of the supercapacitor, 
instead of its intrinsic capacitive performance. Therefore, power density based on discharge in equation (13) should be avoided, and equation (12) should be used when it is possible.

\section{Conclusion}

A method for reporting power and energy density for supercapacitor research is proposed, such that objective comparisons can be made between different research studies as well as commercial products. In addition to conventionally reported metrics such as ESR and capacitance, the extensive property (mass and volume) of the sample is also required. Power and energy densities using this new approach are calculated for some widely used commercial supercapacitors; both results are found to be higher than some recent published values in general. Therefore, for laboratory prototypes, higher capacitance and lower ESR are needed to compete with commercial supercapacitors. Last, a commonly used approach in literature for power density computing is discussed and shown to be potentially erroneous under circumstances in which the arbitrarily chosen galvanostatic current exceeds the intrinsic capabilities of the sample under test.

\section{References}

[1] Burke A F 2007 Proc. IEEE 95806

[2] Pan N and Zhang S L 2015 Adv. Energy Mater. 51401401

[3] Simon P, Gogotsi Y and Dunn B 2014 Science 3431210

[4] Xiong F P, Meng C Z, Reifenberger R, Irazoqui P R and Fisher T 2014 Adv. Energy Mater. 41300515

[5] ACTE UK 2018 http://www.acte-uk.com/technologies/ ultracapacitors

[6] Maxwell Technologies 2018 http://www.maxwell.com/ products/ultracapacitors/16v-small-modules

[7] Levie R 1964 Electrochim. Acta 91231

[8] GreenCap supercapacitor datasheet http://www.masters.com. $\mathrm{pl} /$ files/ds/samwha/samwha-green-cap.pdf

[9] AVX supercapacitor datasheet https://www.mouser.com/ds/2/ 40/AVX-SCC-3.0V-1128335.pdf

[10] Maxwell Technologies datasheet https://www.mouser.com/ds/ 2/257/Maxwell_HCSeries_DS_1013793-9-1179788.pdf

[11] Xiong G P, He P G, Wang D N, Zhang Q Q, Chen T F and Fisher T 2016 Adv. Energy Mater. 265460

[12] Lin T Q, Chen I W, Liu F X, Yang C Y, Bi H, Xu F F et al 2015 Science 3501508

[13] Salunkhe R, Young C, Tang J, Takei T, Ide Y, Kobayashi N et al 2016 Chem. Commun. 524764

[14] Kotz R and Carlen M 2000 Electrochim. Acta 452483

[15] Mondal S, Rana U and Malik S 2015 Chem. Commun. 5112365

[16] Acerce M, Voiry D and Chhowalla M 2015 Nat. Nanotechnol. 10313 